http://economix.fr

Are the Laffer curve and the Green Paradox mutually exclusive?

Document de Travail

Stefano Bosi

Working Paper 2016-09 


\title{
Are the Laffer curve and the Green Paradox mutually exclusive?
}

\author{
Stefano BOSI \\ David DESMARCHELIER \\ EPEE, University of Evry \\ ECONOMIX, University of Paris 10
}

February 15, 2016

\begin{abstract}
To study the relationship between a Laffer Curve and the Green Paradox, we consider a Ramsey model with endogenous labor supply, where pollution increases the consumption demand (compensation effect). In the long run, the conditions for a Laffer curve and the Green Paradox are mutually exclusive: the curve exists under a weak compensation effect while the paradox under a strong effect. In the short run, limit cycles arise only if a Laffer curve exists but never occur in the case of Green Paradox.

Keywords: Ramsey model; Pollution; Laffer Curve; Green Paradox; Hopf bifurcation.

JEL Classificaftion: E32, O44.
\end{abstract}




\section{Introduction}

The Laffer Curve (henceforth, LC) is a well-known paradox discovered by a Muslim scholar a long time ago ${ }^{1}$ but popularized by Arthur Laffer in the seventies (Wanniski, 1978): the tax revenue is an inverted U-shaped function of the tax rate and two different tax rates yield the same revenue. Since, a number of theoretical models either static or dynamic have been published. The introduction of a LC in Ramsey models dates back to the nineties (among others, Irland, 1994; Schmitt-Grohé and Uribe, 1997; Trabandt and Uhlig, 2011; Nourry et al., 2013). Different kinds of taxes generate the LC: for instance, a higher consumption tax lowers the relative leisure price, labor supply and consumption demand in turn, that is the tax revenue in the end.

The Green Paradox (henceforth, GP) is another surprising result of environmental literature identified by Sinn (2008): the announce of a greener tax tomorrow induces the owners of fossil fuels to increase their extraction today and the global warming in the end. Since, theoretical literature on the GP flourished either in partial equilibrium (Sinn, 2008, 2012; Gerlagh, 2011) or in general equilibrium models (Van der Meijden et al., 2015; Eichner and Pethig, 2011; Van der Ploeg and Withagen, 2012; Grafton et al., 2012).

Our paper addresses the issue of compatibility of these paradoxes under a green taxation. Economic literature treats these phenomena separately. We may wonder whether they are mutually exclusive through the fiscal mechanism.

We consider a Ramsey economy with endogenous labor supply where the pollution externalities generated by a consumption activity increase the marginal utility of consumption (compensation effect). The government levies a consumption tax to finance depollution activities. We focus on the long run (comparative statics) and the short run (local dynamics).

At the steady state, along the downward-sloping branch of a LC, a higher consumption tax implies a lower tax revenue. However, the LC may fail to exist if we introduce pollution in the model. Since Michel and Rotillon (1995), it is known that pollution may increase the consumption demand (through the so-called compensation effect). If the tax revenue finances depollution expenditures, pollution increases and consumption as well under a compensation effect. Thereby, the higher tax revenue cancels out the Laffer effect. We show that a weak compensation effect leads to a LC while a strong compensation effect leads to a GP. These sufficient conditions are incompatible. We show also that taxation is welfare-improving beyond a critical point: (1) under a weak compensation effect (sufficient for a LC), if households overevaluate the environmental quality or (2) under a strong compensation effect (sufficient for the GP), if households underevaluate the environmental quality.

In the short run, a compensation effect may give rise to a limit cycle near the steady state of a Ramsey economy (Heal, 1982). ${ }^{2}$ We compare conditions

\footnotetext{
1 "It should be known that at the beginning of a dynasty, taxation yields a large revenue from small assessments. At the end of the dynasty, taxation yields a small revenue from large assessments" (Ibn Khaldun, 1377, p. 230).

${ }^{2}$ Bosi and Desmarchelier (2015) find opposite results in the case of an environmental
} 
for limit cycles (through a Hopf bifurcation) with those for a LC or the GP. We find that the occurrence of limit cycles implies the existence of a LC but these cycles are ruled out in the case of GP.

The rest of the paper is organized as follows. Sections 2 to 5 introduce the fundamentals. Section 6 focuses on the general equilibrium, while sections 7 and 8 on the steady state. Equilibrium transition and bifurcations are considered in section 9 . The case with constant elasticities is studied in section 10 . Section 11 concludes. All the technical details (proofs) are gathered in the appendix.

\section{Firms}

A representative firm produces a single output. A constant returns to scale technology is represented by an aggregate production function:

$$
Y(t)=F(K(t), L(t))
$$

where $K(t)$ and $L(t)$ are the aggregate demands for capital and labor at time $t$. For notational parsimony, the time argument $t$ will be omitted in the following.

Assumption 1 The production function $F: \mathbb{R}_{+}^{2} \rightarrow \mathbb{R}_{+}$is $C^{1}$, homogeneous of degree one, strictly increasing and concave. Inada conditions hold.

The firm chooses the amount of capital and labor to maximize the profit taking the real interest rate $r$ and the real wage $w$ as given. The program $\max _{K, L}[F(K, L)-r K-w L]$ is correctly defined under Assumption 1 and the first-order conditions write:

$$
r=f^{\prime}(k) \equiv r(k) \text { and } w=f(k)-k f^{\prime}(k) \equiv w(k)
$$

where $f(k) \equiv F(k, 1)$ is the average productivity and $k \equiv K / L$ denotes the capital intensity at time $t$. We introduce the capital share in total income and the elasticity of capital-labor substitution:

$$
\alpha(k) \equiv \frac{k f^{\prime}(k)}{f(k)} \text { and } \sigma(k)=\alpha(k) \frac{w(k)}{k w^{\prime}(k)}
$$

In addition, we determine the elasticities of factor prices:

$$
\frac{k r^{\prime}(k)}{r(k)}=-\frac{1-\alpha(k)}{\sigma(k)} \text { and } \frac{k w^{\prime}(k)}{w(k)}=\frac{\alpha(k)}{\sigma(k)}
$$

\section{Household}

The household earns a capital income $r h$ and a labor income $w l$ where $h$ and $l$ denote the individual wealth and labor supply at time $t$. The household pays

Kuznets effect: limit cycles arise under a distaste effect, but they don't under a compensation effect. 
a pollution $\operatorname{tax} \tau$ on consumption to the public authority. Thus, the household consumes and saves her income according to the budget constraint

$$
(1+\tau) c+\dot{h} \leq(r-\delta) h+w l
$$

where $\dot{h}$ denotes the time-derivative of wealth. The gross investment includes the capital depreciation at the rate $\delta$.

For the sake of simplicity, the population of consumers-workers is constant over time and normalized to one: $N=1$. Such a normalization implies $L=$ $N l=l, K=N h=h$ and $h=K / N=k l$.

In the following, $P$ will denote the stock of pollution (aggregate externality).

Assumption 2 Preferences are rationalized by the utility function $u(c, P)-$ $v(l)$. First and second-order restrictions hold on the sign of derivatives: $u_{c}>0$, $u_{P}<0, v_{l}>0$ and $u_{c c}<0, v_{l l}>0$ jointly with the limit conditions: $\lim _{c \rightarrow 0^{+}} u_{c}=\infty$ and $\lim _{l \rightarrow 0^{+}} v_{l}=0$.

Assumption 2 does not impose any restriction on the sign of the crossderivative $u_{c P} \lesseqgtr 0$. Following Michel and Rotillon (1995), the household's preferences exhibit a distaste effect (compensation effect) when pollution decreases (increases) the marginal utility of consumption. If the household enjoys to consume in a pleasant environment, a higher pollution level lowers her consumption demand $\left(u_{c P}<0\right)$ giving rise to a distaste effect (Michel and Rotillon, 1995). Conversely, the household may decide to increase her consumption demand to compensate the utility loss due to a higher pollution level $\left(u_{c P}>0\right)$ : a compensation effect takes place (Michel and Rotillon, 1995).

It is valuable to introduce first and second-order preference elasticities

$$
\begin{gathered}
\varepsilon_{c} \equiv \frac{c u_{c}}{u}, \varepsilon_{P} \equiv \frac{P u_{P}}{u} \text { and } \varepsilon_{l} \equiv \frac{l v_{l}}{v} \\
\varepsilon_{c c} \equiv \frac{c u_{c c}}{u_{c}}, \varepsilon_{c P} \equiv \frac{P u_{c P}}{u_{c}} \text { and } \varepsilon_{l l} \equiv \frac{l v_{l l}}{v_{l}}
\end{gathered}
$$

$-1 / \varepsilon_{c c}$ is the consumption elasticity of intertemporal substitution while $\varepsilon_{c P}$ captures the effects of pollution on the marginal utility of consumption. According to Assumption 2, $\varepsilon_{c c}<0$. In terms of elasticity, the compensation effect writes $\varepsilon_{c P}>0$.

In a Ramsey model, the representative household maximizes an intertemporal utility functional $\int_{0}^{\infty} e^{-\rho t}[u(c, P)-v(l)] d t$ under the budget constraint (3) where $\rho>0$ denotes the rate of time preference. This program is correctly defined under Assumption 2.

Proposition 1 The first-order conditions result in a static consumption-leisure relation

$$
\frac{u_{c}(c, P)}{1+\tau}=\lambda=\frac{v_{l}(l)}{w}
$$

a dynamic Euler equation $\dot{\lambda}=\lambda(\rho+\delta-r)$ and the budget constraint (3), now binding: $\dot{h}=(r-\delta) h+w l-(1+\tau) c$, jointly with the transversality condition 
$\lim _{t \rightarrow \infty} e^{-\rho t} \lambda h=0$, where $\lambda$ denotes the multiplier associated to the budget constraint.

Proof. See Appendix.

\section{Government}

An environment-oriented government spends the tax revenue to finance depollution through an abatement effort (maintenance $m$ ) according to a balanced budget rule:

$$
m=\tau C
$$

For simplicity, the population is normalized to one. Thus, $C=N c=c$ and budget (7) writes in intensive terms: $\tau c=m$.

\section{Pollution}

The aggregate stock of pollution $P$ is a pure externality coming from consumption $(C)$. In addition, the government takes care of depollution through the abatement expenditures $m$. To take things as simple as possible, we assume a linear process:

$$
\dot{P}=-a P+b C-\gamma m
$$

$a \geq 0, b \geq 0$ and $\gamma \geq 0$ capture respectively the natural rate of pollution absorption, the environmental impact of consumption and the pollution abatement efficiency. Because $N=1$, the process of pollution accumulation (8) writes in intensive terms: $\dot{P}=-a P+b c-\gamma m$.

\section{Equilibrium}

At the equilibrium, all markets clear. Under Assumption 2, we apply the implicit function theorem to the static relation (6) to obtain $c \equiv c(\lambda, P)$ and $l \equiv l(\lambda, k)$ with:

$$
\begin{aligned}
\frac{\lambda}{c} \frac{\partial c}{\partial \lambda} & =\frac{1}{\varepsilon_{c c}} \text { and } \frac{P}{c} \frac{\partial c}{\partial P}=-\frac{\varepsilon_{c P}}{\varepsilon_{c c}} \\
\frac{k}{l} \frac{\partial l}{\partial k} & =\frac{\alpha(k)}{\sigma(k)} \frac{1}{\varepsilon_{l l}} \text { and } \frac{\lambda}{l} \frac{\partial l}{\partial \lambda}=\frac{1}{\varepsilon_{l l}}
\end{aligned}
$$

This implies that:

$$
\frac{\dot{h}}{h}=\left[1+\frac{\alpha(k)}{\sigma(k)} \frac{1}{\varepsilon_{l l}}\right] \frac{\dot{k}}{k}+\frac{1}{\varepsilon_{l l}} \frac{\dot{\lambda}}{\lambda}
$$


Proposition 2 Equilibrium dynamics are represented by the following system:

$$
\begin{aligned}
& \dot{\lambda}=g_{1}(\lambda, k, P) \equiv[\rho+\delta-r(k)] \lambda \\
& \dot{k}=g_{2}(\lambda, k, P) \equiv \frac{[r(k)-\delta] k+w(k)-(1+\tau) \frac{c(\lambda, P)}{l(\lambda, k)}-\frac{[\rho+\delta-r(k)] k}{\varepsilon_{l l}}}{1+\frac{\alpha(k)}{\sigma(k)} \frac{1}{\varepsilon_{l l}}} \\
& \dot{P}=g_{3}(\lambda, k, P) \equiv-a P+(b-\gamma \tau) c(\lambda, P)
\end{aligned}
$$

Proof. (12) is the Euler equation of Proposition 1, (13) is the equilibrium budget constraint of Proposition 1 where we have replaced (11). (14) is the pollution accumulation process (8) where we have replaced the balanced budget rule (7).

Equations (12), (13) and (14) form a dynamic system. This system has two backward variables $(k$ and $P$ ) and one jump variable $(\lambda)$.

\section{Steady state}

At the steady state, $\dot{\lambda}=\dot{k}=\dot{P}=0$, the system (12)-(13)-(14) gives:

$$
\begin{aligned}
r(k) & =\rho+\delta \\
\frac{c(\lambda, P)}{l(\lambda, k)} & =\frac{\rho k+w(k)}{1+\tau} \\
P & =\frac{b-\gamma \tau}{a} c(\lambda, P)
\end{aligned}
$$

Equation (15) is the MGR of the standard Ramsey model. Assumption 1 ensures the uniqueness of $k^{*}$ :

$$
k^{*}=r^{-1}(\rho+\delta)>0
$$

In the following of this section, for simplicity, $k$ and $w$ will denote $k^{*}$ and $w\left(k^{*}\right)$.

Applying the Implicit Function Theorem to equation (17) gives $\lambda=\lambda(P)$ with

$$
\frac{P}{\lambda} \frac{d \lambda}{d P}=\varepsilon_{c c}+\varepsilon_{c P}<0 \text { iff } \varepsilon_{c P}<-\varepsilon_{c c}
$$

Let now

$$
\mu(P) \equiv \frac{c(\lambda(P), P)}{l(\lambda(P), k)} \text { and } \theta \equiv \frac{P}{\mu} \frac{\partial \mu}{\partial P}
$$

Thus, the existence and uniqueness of the steady state for this economy depend upon the number of positive $P^{*}$ satisfying equation

$$
\mu(P)=\frac{\rho k+w}{1+\tau}(>0)
$$

Assumption 3 Let $\tau<b / \gamma$ in the sequel.

This assumption ensures that $P$ is always positive (see equation (17)). 
Proposition 3 (existence and uniqueness) Let Assumptions 1, 2 and 3 hold. A steady state exists if

$$
\lim _{P \rightarrow 0} \mu(P)<\frac{\rho k+w}{1+\tau}<\lim _{P \rightarrow \infty} \mu(P)
$$

Moreover, if $\varepsilon_{c P}<-\varepsilon_{c c}$, the steady state is unique.

Proof. See the Appendix.

Interestingly, $\varepsilon_{c P}<-\varepsilon_{c c}$ is always verified when preferences are characterized by a distaste effect (or a weak compensation effect). Later in this paper, we will discuss the existence of a steady state by considering isoelastic functional forms.

\section{Comparative statics}

It is worthy to consider the impact of the tax rate on the main variables. Nevertheless, our general formulation doesn't avoid the complicated case where elasticities (5) are functions of $\tau$. The adoption of isoelastic functional forms will rule out this unpleasant situation and will allow us to provide unambiguous explicit conditions for the existence of both the LC and the GP.

From (15), we find

$$
\frac{\tau}{k} \frac{\partial k}{\partial \tau}=0
$$

Then, the tax rate has no effect on the capital level of steady state. This is not surprising in a decentralized economy: the representative household doesn't internalize the pollution externality and pollution doesn't appear in her Euler equation, that is, at the steady state, the MGR, and doesn't affect the capital intensity in turn.

Focus on equations (16) and (17):

$$
l=\frac{(1+\tau) c}{\rho k+w} \text { and } P=\frac{b-\gamma \tau}{a} c
$$

Replacing them in the arbitrage between consumption and labor supply (6), we find

$$
w u_{c}\left(c, \frac{b-\gamma \tau}{a} c\right)=(1+\tau) v_{l}\left(\frac{(1+\tau) c}{\rho k+w}\right)
$$

Applying the Implicit Function Theorem to (20), we obtain $c=\tilde{c}(\tau)$ with elasticity

$$
\pi(\tau) \equiv \frac{\tau \tilde{c}^{\prime}(\tau)}{\tilde{c}(\tau)}=\frac{\tau}{1+\tau} \frac{1+\varepsilon_{l l}+\varepsilon_{c P} \frac{\gamma+\gamma \tau}{b-\gamma \tau}}{\varepsilon_{c c}+\varepsilon_{c P}-\varepsilon_{l l}}
$$

The effect of an increase in $\tau$ on long-run consumption is ambiguous and depends upon the magnitude of the pollution effect on the marginal utility of consumption. For instance, assume that preferences display a compensation effect $\left(\varepsilon_{c P}>0\right)$. 
Under a weak effect $\left(0<\varepsilon_{c P}<\varepsilon_{l l}-\varepsilon_{c c}\right)$, we have $\pi(\tau)<0$. This negative impact is not surprising and advocates for the existence of a LC. Indeed, a higher consumption tax lowers the relative leisure price which reduces labor supply and consumption demand, inducing a lower tax revenue in turn. In section 10.3, we will illustrate the possibility of LC.

Conversely, under an excessive compensation effect $\left(\varepsilon_{c P}>\varepsilon_{l l}-\varepsilon_{c c}>0\right)$, we have $\pi(\tau)>0$. Of course, in this case, the LC vanishes. Such an impossibility will be also considered in section 10.3.

However, such surprising outcome deserves an economic interpretation. The above argument for the existence of a LC holds without pollution effect on consumption demand or, by continuity, under a low pollution effect (namely, $0<\varepsilon_{c P}<\varepsilon_{l l}-\varepsilon_{c c}$ ). It does not take into account the general interplay between tax revenue, pollution level and consumption demand, namely the case of an excessive pollution effect on consumption demand $\left(\varepsilon_{c P}>\varepsilon_{l l}-\varepsilon_{c c}>0\right)$. An increase in the green consumption tax rate implies a lower tax revenue and a lower depollution leading to a higher pollution level. Since preferences exhibit a strong compensation effect, the consumption demand increases and the tax revenue as well preventing the LC existence.

Thus, LC fails to exist because of the positive relation between the green consumption tax rate and the pollution level (our definition of GP revisits that introduced by Sinn (2008)). To understand this point, we need to know the effect of the green tax rate on the pollution level of steady state.

We observe that

$$
\tilde{l}(\tau) \equiv \frac{1+\tau}{\rho k+w} \tilde{c}(\tau)
$$

and, thus,

$$
\frac{\tau \tilde{l}^{\prime}(\tau)}{\tilde{l}(\tau)}=\pi(\tau)+\frac{\tau}{1+\tau}
$$

This implies

$$
\tilde{P}(\tau) \equiv \frac{b-\gamma \tau}{a} \tilde{c}(\tau)
$$

with elasticity

$$
\frac{\tau \tilde{P}^{\prime}(\tau)}{\tilde{P}(\tau)}=\pi(\tau)-\frac{\gamma \tau}{b-\gamma \tau}
$$

We remark that $\pi(\tau)>0$ is a necessary condition for a positive effect of $\tau$ on $P(\mathrm{GP})$. As seen above, $\pi(\tau)>0$ excludes the existence of a LC. That is, the occurrence of the GP seems to exclude the LC existence. More economic intuition will be provided in section 10.3.

These computations shed light on the welfare effects of a green tax. Because of the representative agent, the welfare function is given by her utility function.

At the steady state, this function becomes:

$$
\begin{aligned}
\tilde{W}(\tau) & \equiv \int_{0}^{\infty} e^{-\rho t}[u(\tilde{c}(\tau), \tilde{P}(\tau))-v(\tilde{l}(\tau))] d t \\
& =\frac{1}{\rho}[u(\tilde{c}(\tau), \tilde{P}(\tau))-v(\tilde{l}(\tau))]
\end{aligned}
$$


Let

$$
\pi^{*} \equiv \frac{\frac{\varepsilon_{P}}{\varepsilon_{c}} \frac{\gamma \tau}{b-\gamma \tau}+\frac{\tau}{1+\tau} \frac{(1-\alpha)(\delta+\rho)}{(1-\alpha) \delta+\rho}}{\frac{\varepsilon_{P}}{\varepsilon_{c}}+\frac{\alpha \rho}{(1-\alpha) \delta+\rho}}
$$

with

$$
\varepsilon_{c} \equiv \frac{c u_{c}}{u}, \varepsilon_{P} \equiv \frac{P u_{P}}{u} \text { and } \varepsilon_{l} \equiv \frac{l v_{l}}{v}
$$

Notice that $\varepsilon_{P} / \varepsilon_{c}<0$. We have the following proposition.

Proposition 4 Let Assumptions 1, 2, and 3 hold.

(1) If $\varepsilon_{P} / \varepsilon_{c}<-\alpha \rho /[(1-\alpha) \delta+\rho]$, taxation is welfare-improving $\left(\tilde{W}^{\prime}(\tau)>\right.$ $0)$ if and only if $\pi(\tau)<\pi^{*}$.

(2) If $-\alpha \rho /[(1-\alpha) \delta+\rho]<\varepsilon_{P} / \varepsilon_{c}<0$, taxation is welfare-improving $\left(\tilde{W}^{\prime}(\tau)>\right.$ $0)$ if and only if $\pi(\tau)>\pi^{*}$.

Proof. See Appendix.

We notice that both $\pi(\tau)$ and $\pi^{*}$ depend on $\tau$. Explicit conditions will be provided in section 10.4 .

The elasticity ratio $\varepsilon_{P} / \varepsilon_{c}$ captures both the slope of the indifference curve and the household's relative preference for environmental quality with respect to consumption. Taking into account previous results about the effects of $\tau$ on $c$ and $P$, Proposition 4 shows that the welfare effect of the green consumption tax depends upon two elements: (1) the existence of a LC or of a GP (depending on the sign of $\pi(\tau)$ ) and (2) households' relative preference for environmental quality with respect to consumption (depending on the magnitude of $\varepsilon_{P} / \varepsilon_{c}$ ). In section 10.4, we will deepen these considerations.

\section{Local dynamics}

Several contributions point out that pollution may promote macroeconomic instability. Fernandez et al. (2012) and Itaya (2008) show that a large positive pollution effect on the marginal utility of consumption (compensation effect) may lead to local indeterminacy in a simple Ramsey economy with endogenous labor supply when pollution comes from capital accumulation. More recently, Bosi et al. (2015) find that a positive pollution effect on the marginal disutility of labor supply is also a source of endogenous fluctuations. Our model is close to Fernandez et al. (2012) but differs in three respects: (1) pollution is a stock variable, (2) pollution comes from consumption and (3) a green consumption tax is considered. Since we analyze a Ramsey economy with endogenous labor supply and a consumption tax, our model is also close to Nourry et al. (2013) but: (1) pollution matters and (2) the consumption tax rate is exogenous.

According to Heal (1982), Itaya (2008) and Fernandez et al. (2012), the compensation effect is a source of endogenous cycles. In the previous section, we have seen that the potential occurrence of both a LC or the GP rests on the magnitude of this effect. It is valuable to compare the parameter ranges 
where endogenous cycles take place and where a LC or the GP occurs. In the following, we tackle this question.

We linearize system (12)-(14) around the steady state to grasp the equilibrium stability properties. For simplicity, in this section, we consider the case of a constant elasticity $\varepsilon_{l l}=\xi$ and of a Cobb-Douglas production function: $f(k) \equiv A k^{\alpha}$, that is, $\alpha(k)=\alpha$ and $\sigma(k)=1$. Equation (13) writes

$$
\dot{k}=\frac{\xi}{\alpha+\xi}\left([r(k)-\delta] k+w(k)-(1+\tau) \frac{c(\lambda, P)}{l(\lambda, k)}-\frac{[\rho+\delta-r(k)] k}{\xi}\right)
$$

The Jacobian matrix $J$ writes

$$
\begin{aligned}
J & \equiv\left[\begin{array}{lll}
\frac{\partial g_{1}}{\partial \lambda} & \frac{\partial g_{1}}{\partial k} & \frac{\partial g_{1}}{\partial P} \\
\frac{\partial g_{2}}{\partial \lambda} & \frac{\partial g_{2}}{\partial k} & \frac{\partial g_{2}}{\partial P} \\
\frac{\partial g_{3}}{\partial \lambda} & \frac{\partial g_{3}}{\partial k} & \frac{\partial g_{3}}{\partial P}
\end{array}\right] \\
& =\left[\begin{array}{ccc}
0 & (1-\alpha)(\rho+\delta) \frac{\lambda}{k} & 0 \\
-\frac{\xi}{\alpha} \frac{\rho+(1-\alpha) \delta}{\alpha+\xi} \frac{k}{\lambda}\left(\frac{1}{\varepsilon_{c c}}-\frac{1}{\xi}\right) & \rho & \frac{\xi}{\alpha} \frac{\rho+(1-\alpha) \delta}{\alpha+\xi} \frac{k}{P} \frac{\varepsilon_{c P}}{\varepsilon_{c c}} \\
a \frac{P}{\lambda} \frac{1}{\varepsilon_{c c}} & 0 & -a\left(1+\frac{\varepsilon_{c P}}{\varepsilon_{c c}}\right)
\end{array}\right]
\end{aligned}
$$

We compute the trace, the sum of minors of order two and the determinant.

$$
\begin{aligned}
T & =\rho-a\left(1+\frac{\varepsilon_{c P}}{\varepsilon_{c c}}\right) \\
S & =\frac{(1-\alpha)(\rho+\delta)[\rho+(1-\alpha) \delta]}{\alpha(\alpha+\xi)}\left(\frac{\xi}{\varepsilon_{c c}}-1\right)-a \rho\left(1+\frac{\varepsilon_{c P}}{\varepsilon_{c c}}\right) \\
D & =a(1-\alpha)(\rho+\delta) \frac{\xi}{\alpha} \frac{\rho+(1-\alpha) \delta}{\alpha+\xi}\left[\frac{1}{\xi}\left(1+\frac{\varepsilon_{c P}}{\varepsilon_{c c}}\right)-\frac{1}{\varepsilon_{c c}}\right]
\end{aligned}
$$

We observe that

$$
\begin{aligned}
T & <0 \Leftrightarrow 1+\frac{\varepsilon_{c P}}{\varepsilon_{c c}}>\frac{\rho}{a}(>0) \\
S & <0 \Leftrightarrow 1+\frac{\varepsilon_{c P}}{\varepsilon_{c c}}>\frac{(1-\alpha)(\rho+\delta)[\rho+(1-\alpha) \delta]}{a \rho \alpha(\alpha+\xi)}\left(\frac{\xi}{\varepsilon_{c c}}-1\right)(<0) \\
D & <0 \Leftrightarrow 1+\frac{\varepsilon_{c P}}{\varepsilon_{c c}}<\frac{\xi}{\varepsilon_{c c}}(<0)
\end{aligned}
$$

We will see in section 10.5 that $T, D$ and $S$ are not functions of $\tau$ in the isoelastic case: $\tau$ has no effect on the local stability properties in this case because the representative household adjusts her consumption and labor supply.

The analytical study of a three-dimensional dynamic system is not usual in economics. As in Bosi and Desmarchelier (2015), it is indispensable to introduce a general methodology to characterize local bifurcations and indeterminacy in the case of three-dimensional dynamics with two predetermined variables (sections 9.1, 9.2, 9.3 and 9.4). However, because elasticities (5) are in general complicated, we will apply our general methodology to the simples isoelastic case in section 10.5 . 


\subsection{Bifurcations}

In continuous time, a local bifurcation generically arises when the real part of an eigenvalue $\lambda(p)$ of the Jacobian matrix crosses zero in response to a change in a parameter $p$. Denoting by $p^{*}$ the critical parameter value of bifurcation, we get generically two cases: (1) when a real eigenvalue crosses zero: $\lambda\left(p^{*}\right)=0$, the system undergoes a saddle-node bifurcation (either an elementary saddlenode or a transcritical or a pitchfork bifurcation depending on the number of steady states), (2) when the real part of two complex and conjugate eigenvalues $\lambda(p)=a(p) \pm i b(p)$ crosses zero, the system undergoes a Hopf bifurcation. More precisely, in the second case, we require $a\left(p^{*}\right)=0$ and $b(p) \neq 0$ in a neighborhood of $p^{*}$ (see Bosi and Ragot, 2011, p. 76).

The occurrence of a saddle-node bifurcation (elementary saddle-node, transcritical, pitchfork) requires a multiplicity of steady states. In our model, the steady state is unique (Proposition 3). Thus, we leave aside the theory of elementary saddle-node bifurcations to focus exclusively on a general approach to Hopf bifurcations in the case of three-dimensional dynamic systems and on the occurrence of limit cycles.

We eventually observe that system (12)-(14) is three-dimensional with two predetermined variables $(k$ and $P$ ) and one jump variable $(\lambda)$. Thus, multiple equilibria (local indeterminacy) arise when the three eigenvalues of the Jacobian matrix (24) evaluated at the steady state have negative real parts: either $\lambda_{1}, \lambda_{2}, \lambda_{3}<0$ or $\operatorname{Re} \lambda_{1}, \operatorname{Re} \lambda_{2}<0$ and $\lambda_{3}<0$.

\subsection{Saddle-node bifurcation}

Proposition 5 If $\varepsilon_{c P}<-\varepsilon_{c c}$, then any saddle-node bifurcation (elementary saddle node, transcritical, pitchfork) is ruled out.

Proof. Saddle-node bifurcations require the existence of multiple steady states. If $\varepsilon_{c P}<-\varepsilon_{c c}$, then the steady state is unique (Proposition 3 ).

\subsection{Hopf bifurcation}

This bifurcation generates limit cycles either attractive (supercritical) or repulsive (subcritical).

Reconsider the Jacobian matrix $J$ and its determinant, sum of minors of order two and trace: $D=\lambda_{1} \lambda_{2} \lambda_{3}, S=\lambda_{1} \lambda_{2}+\lambda_{1} \lambda_{3}+\lambda_{2} \lambda_{3}$ and $T=\lambda_{1}+\lambda_{2}+\lambda_{3}$. A Hopf bifurcation occurs when the real part of two complex and conjugate eigenvalues $\lambda(p)=a(p) \pm i b(p)$ crosses zero. More precisely, we require $a\left(p^{*}\right)=$ 0 and $b(p) \neq 0$ in a neighborhood of $p^{*}$ (see Bosi and Ragot, 2011, p. 76).

Proposition 6 (Hopf bifurcation) In the case of a three-dimensional system, a Hopf bifurcation generically arises if and only if $D=S T$ and $S>0$.

Proof. See Appendix.

Corollary 7 If $\varepsilon_{c P}<-\varepsilon_{c c}$, Hopf bifurcations are ruled out. 
Proof. If $\varepsilon_{c P}<-\varepsilon_{c c}$, then $S<0$. According to Proposition 6, there is no room for Hopf bifurcations.

\subsection{Local determinacy}

In our economy, there are two predetermined variables $(k$ and $P$ ) and a jump variable $(\lambda)$. As seen above, indeterminacy requires three eigenvalues with negative real parts: either $\lambda_{1}, \lambda_{2}, \lambda_{3}<0$ or $\operatorname{Re} \lambda_{1}, \operatorname{Re} \lambda_{2}<0$ and $\lambda_{3}<0$.

Proposition 8 (local determinacy) If $\varepsilon_{c P}<-\varepsilon_{c c}$, the equilibrium is locally unique.

Proof. If $\varepsilon_{c P}<-\varepsilon_{c c}$, then $D>0$. If the eigenvalues are real, one eigenvalue is positive. Then, local determinacy. If $\lambda_{1}$ and $\lambda_{2}$ are nonreal and conjugated, then $\lambda_{1} \lambda_{2}>0$ and $D=\lambda_{1} \lambda_{2} \lambda_{3}>0$ imply the positivity of the third eigenvalue. Hence, local determinacy.

Proposition 9 If all the eigenvalues are real, the equilibrium is locally indeterminate if and only if $D, T<0$ and $S>0$.

Proof. See Appendix.

\section{Isoelastic case}

We cannot capitalize on our general methodology if the elasticities (5) are mutually dependent and dependent on $\tau$. Comparative statics and local dynamics are of little use without explicit results. In order to find these results and provide clear-cut interpretations we need to consider explicit functional forms.

\subsection{Fundamentals}

Focus on widely used isoelastic functional forms:

$$
\begin{aligned}
u(c, P) & \equiv \frac{\left(c P^{-\eta}\right)^{1-\varepsilon}}{1-\varepsilon} \\
v(l) & \equiv v \frac{l^{1+\xi}}{1+\xi} \\
f(k) & \equiv A k^{\alpha}
\end{aligned}
$$

(25), (26) and (27) implies $\sigma=1$ and

$$
\left[\begin{array}{ccc}
\varepsilon_{c} & \varepsilon_{P} & \varepsilon_{l} \\
\varepsilon_{c c} & \varepsilon_{c P} & \varepsilon_{l l}
\end{array}\right]=\left[\begin{array}{ccc}
1-\varepsilon & -\eta(1-\varepsilon) & 1+\xi \\
-\varepsilon & \eta(\varepsilon-1) & \xi
\end{array}\right]
$$

Isoelastic specifications (25), (26) and (27) imply that elasticities (5) become parametric and, surprisingly, don't depend on $\tau$. 


\subsection{Steady state}

Functions (25), (26) and (27) imply also the existence of a unique steady state:

$$
\begin{aligned}
\lambda & =\frac{P^{\varepsilon \eta-\varepsilon-\eta}}{1+\tau}\left(\frac{b-\gamma \tau}{a}\right)^{\varepsilon} \\
k & =\left(\frac{\alpha A}{\rho+\delta}\right)^{\frac{1}{1-\alpha}} \\
P & =\left[\frac{w / v}{1+\tau}\left(\frac{\rho k+w}{1+\tau}\right)^{\xi}\left(\frac{b-\gamma \tau}{a}\right)^{\varepsilon+\xi}\right]^{\frac{1}{\xi+\varepsilon+\eta-\varepsilon \eta}}
\end{aligned}
$$

with $w=(1-\alpha) A k^{\alpha}$. Moreover,

$$
\begin{aligned}
c & =\frac{a}{b-\gamma \tau} P \\
l & =\frac{1+\tau}{\rho k+w} \frac{a}{b-\gamma \tau} P
\end{aligned}
$$

Isoelastic functional forms lead to a unique steady state even if conditions of Proposition (3) are not met. The explicit solution for the steady state allow us to compute the long-run impact of a green consumption tax. As we have seen in section 8, a LC or the GP are possible. The next section provides sufficient conditions for their existence.

\subsection{Laffer curve and Green Paradox}

In order to shed light on the relation between LC and GP, we compute the effect of $\tau$ on the steady state. We will give conditions under which the green tax rate $(\tau)$ and the tax revenue $(m)$ are related through an inverted U-shaped curve (LC) and conditions under which $\tau$ and the pollution level $(P)$ are linked through a positive relation (GP).

According to (21), the equilibrium consumption depends on the tax rate: $c=\tilde{c}(\tau)$, and the tax receipts as well: $m=\tau \tilde{c}(\tau) \equiv \tilde{m}(\tau)$.

We know from equation (21) the elasticity

$$
\pi(\tau) \equiv \frac{\tau}{\tilde{c}} \frac{d \tilde{c}}{d \tau}
$$

Solving (20) for $c$, we find explicitly

$$
\tilde{c}(\tau)=\left(\frac{(1-\alpha) A k^{\alpha}\left[\rho k+(1-\alpha) A k^{\alpha}\right]^{\xi}}{v(1+\tau)^{1+\xi}}\left(\frac{b-\gamma \tau}{a}\right)^{\eta(\varepsilon-1)}\right)^{\frac{1}{\xi+\varepsilon+(1-\varepsilon) \eta}}
$$

where $k$ is given by (29) and, finally, $\tilde{m}(\tau) \equiv \tau \tilde{c}(\tau)$ :

$$
\tilde{m}(\tau)=\tau\left(\frac{(1-\alpha) A k^{\alpha}\left[\rho k+(1-\alpha) A k^{\alpha}\right]^{\xi}}{v(1+\tau)^{1+\xi}}\left(\frac{b-\gamma \tau}{a}\right)^{\eta(\varepsilon-1)}\right)^{\frac{1}{\xi+\varepsilon+(1-\varepsilon) \eta}}
$$


The two following propositions summarize some results of comparative statics.

Proposition 10 (existence of LC) Let $0<\eta(\varepsilon-1)<\varepsilon+\xi$. There exists a unique $\tau^{*} \in(0, b / \gamma)$ such that $\partial m / \partial \tau>0$ if and only if $\tau<\tau^{*}$.

Proof. See Appendix.

The existence of a LC is not surprising. Indeed, a higher consumption tax lowers the relative leisure price which gives an incentive to reduce consumption and to supply less labor. Such a situation implies a lower tax revenue which explains the counter-intuitive downward-sloping branch of the LC (see Nourry et al. (2013) among others). Interestingly, proposition 10 no longer holds when preferences display an excessive compensation effect (namely, if $\eta(\varepsilon-1)>\varepsilon+$ $\xi)$. In this case, the LC fails to exist.

Let us give some intuition (see also section 8). On the downward-sloping branch of the LC, a higher green tax rate implies a lower tax revenue entailing a lower depollution and a higher pollution stock in turn. Under an excessive compensation effect, consumption demand increases enough to raise the tax revenue and prevent the existence of a LC. Interestingly, the LC seems to fail because of the positive effect of the green tax rate on the pollution level (GP). That is, the GP seems to rule out the LC. To grasp this point, we compute the effect of $\tau$ on the pollution stock of steady state.

Proposition 11 (existence of GP) Let Assumptions 1, 2 and 3 hold.

$$
\begin{aligned}
& \text { If } \eta(\varepsilon-1)<\varepsilon+\xi \text {, then }(\tau / \tilde{P})(\partial \tilde{P} / \partial \tau)<0 . \\
& \text { If } \eta(\varepsilon-1)>\varepsilon+\xi \text {, then }(\tau / \tilde{P})(\partial \tilde{P} / \partial \tau)>0(G P) .
\end{aligned}
$$

Proof. Notice that $P>0$ requires $\tau \in(0, b / \gamma)$. Replace expression (21) in (23) to verify the proposition.

We observe that, according to notation (31) and Proposition 11, the GP arises when $\varphi<-\xi / \varepsilon<0$.

Corollary 12 Sufficient conditions for LC and GP are mutually exclusive.

Proof. Compare conditions in Proposition 10 and 11.

To the best of our knowledge, the GP literature focuses on fossil fuel extraction $^{3}$ to explain how a higher green tax may increase the pollution level and exacerbate the global warming. Since the GP refers to a positive effect of a green tax on pollution, Proposition 11 points out that an excessive compensation effect $(\eta(\varepsilon-1)>\varepsilon+\xi)$ also promotes the GP.

Interestingly, as soon as the LC disappears, the GP emerges. That is, the LC disappears because a higher consumption tax rate increases the pollution level (GP), under a strong compensation effect, this generates a higher consumption level which implies a higher tax revenue. Thus, in an economy where households'

\footnotetext{
${ }^{3}$ The interested reader is referred to Van der Meijden et al. (2015), Eichner and Pethig (2011), Van der Ploeg and Withagen (2012) and Grafton et al. (2012) among others.
} 
preferences are characterized by a strong (weak) compensation effect, the LC fails to exist (may exist) while the GP may arise (fails to occur).

An environment-oriented government needs to know precisely the consumers' preferences: under a strong compensation effect, a higher green tax rate on consumption may promote a higher pollution level (GP). In this case, the green tax is not suited to clean the environment.

The reader is referred to the end of section 10 for a graphical illustration of LC and GP.

\subsection{Welfare analysis}

We introduce a critical taxation point

$$
\tau^{*} \equiv \frac{b(1-\alpha)(\delta+\rho)-\gamma \eta[(1-\alpha) \delta+\rho]}{\gamma(1-\alpha)(\delta+\rho)+\gamma \eta[(1-\alpha) \delta+\rho]}
$$

and the critical sensitivity to pollution $\eta^{*} \equiv \alpha \rho /[(1-\alpha) \delta+\rho]$.

Proposition 13 Let assumption 1, 2 and 3 hold. Since $\varepsilon>1$ (compensation effect), $\tau$ has a positive welfare effect when $\tau>\tau^{*}$ jointly with:

(1) $\eta>\eta^{*}$ if $\eta(\varepsilon-1)<\varepsilon+\xi$ (sufficient condition for $L C$ ).

(2) $\eta<\eta^{*}$ if $\eta(\varepsilon-1)>\varepsilon+\xi$ (sufficient condition for GP).

Proof. See Appendix.

Let us explain Proposition 13. According to $28, \eta=-\varepsilon_{P} / \varepsilon_{c}$ captures the slope of the indifference curve with utility $u(c, P)$ and the relative preference of environmental quality with respect to consumption.

First, assume that the economy experiences a weak compensation effect $(\eta(\varepsilon-1)<\varepsilon+\xi$, implying LC). According to Propositions 10 and 11, a higher green consumption tax rate implies a lower consumption level and a lower pollution level (higher environmental quality). If the household over-evaluates the environmental quality with respect to consumption $\left(\eta>\eta^{*}\right)$, the utility loss induced by the drop in consumption is over-compensated by the utility gain due to a cleaner environment: the pollution tax turns out to be welfare-improving.

Now, assume that the economy experiences a strong compensation effect $(\eta(\varepsilon-1)>\varepsilon+\xi$, implying GP). By definition of GP, a higher green tax rate increases the pollution level (Proposition 11) and the consumption level in turn (equation 30). If the representative household under-evaluates environmental quality with respect to consumption $\left(\eta<\eta^{*}\right)$, the utility loss induced by the drop in environmental quality is over-compensated by the utility gain induced by the higher consumption level: the pollution tax turns out to be welfare improving.

\subsection{Local dynamics}

Since Heal (1982), it is known that a strong compensation effect can lead to persistent deterministic cycles (Hopf) in a continuous-time Ramsey model. Such 
dynamical phenomena occur near the steady state (29). In this section, we shed light upon the occurrence of cycles and their with the LC and the GP.

Let

$$
\begin{aligned}
\varphi & \equiv 1-\eta \frac{\varepsilon-1}{\varepsilon}=1+\frac{\varepsilon_{c P}}{\varepsilon_{c c}} \\
\psi & \equiv(\rho+\delta) \frac{1-\alpha}{\alpha} \frac{\rho+(1-\alpha) \delta}{\alpha+\xi}>0
\end{aligned}
$$

Consider (14), (38) and (39) to compute $T, S$ and $D$ :

$$
\begin{aligned}
T & =\rho-a \varphi \\
S & =-\psi\left(1+\frac{\xi}{\varepsilon}\right)-a \varphi \rho \\
D & =a \psi\left(\varphi+\frac{\xi}{\varepsilon}\right)
\end{aligned}
$$

Let

$$
\eta_{H} \equiv \frac{\varepsilon\left(1-\varphi_{1}\right)}{\varepsilon-1}
$$

with

$$
\begin{aligned}
& \varphi_{1}=\frac{1}{2 a}\left[\rho-\frac{\xi}{\varepsilon} \frac{\psi}{\rho}-\sqrt{\left(\rho-\frac{\xi}{\varepsilon} \frac{\psi}{\rho}\right)^{2}+4 \psi\left(1+\frac{\xi}{\varepsilon} \frac{a+\rho}{\rho}\right)}\right] \\
& \varphi_{2}=\frac{1}{2 a}\left[\rho-\frac{\xi}{\varepsilon} \frac{\psi}{\rho}+\sqrt{\left(\rho-\frac{\xi}{\varepsilon} \frac{\psi}{\rho}\right)^{2}+4 \psi\left(1+\frac{\xi}{\varepsilon} \frac{a+\rho}{\rho}\right)}\right]
\end{aligned}
$$

Proposition 14 Let $\varepsilon>1$. If

$$
\eta_{H}>\frac{\varepsilon}{\varepsilon-1}\left[1+\frac{\psi}{a \rho}\left(1+\frac{\xi}{\varepsilon}\right)\right]
$$

a limit cycle generically arises through a Hopf bifurcation near the steady state at $\eta=\eta_{H}$.

Proof. A Hopf bifurcation generically requires $S>0$ and $D=S T$, that is

$$
\varphi<-\frac{\psi}{a \rho}\left(1+\frac{\xi}{\varepsilon}\right)
$$

and $\varphi=\varphi_{1}$ or $\varphi_{2}$. Since $\varphi_{1}<0<\varphi_{2}$, the Hopf bifurcation generically arises only at $\varphi=\varphi_{1}$ provided that (36) holds, that is (35).

Corollary 15 (equilibrium determinacy) There is no room for local indeterminacy around the Hopf bifurcation point. 
Proof. A necessary condition for local indeterminacy is $T<0$. At the Hopf bifurcation point, $T=\rho-a \varphi_{1}>0$ because $\varphi_{1}<0$ : an unstable eigenvalue always exists when the Hopf bifurcation occurs.

Lemma $16 D>0$ implies a $L C$, while $D<0$ implies $G P$.

Proof. According to $31, D>0$ if and only if $\eta(\varepsilon-1)<\varepsilon+\xi$. Then $D>0$ implies LC, while $D<0$ implies GP.

Proposition 17 If a Hopf bifurcation occurs, the economy experiences a LC. Conversely, there is no room for a Hopf bifurcation under the GP.

Proof. According to the proof of Proposition 14, at the Hopf bifurcation point, $\varphi=\varphi_{1}<0$, that is, $T=\rho-a \varphi_{1}>0$. From Proposition 6, a Hopf bifurcation generically occurs if and only if $D=S T$ with $S>0$. Thus, $D>0$ becomes a necessary condition to observe a Hopf bifurcation. According to Lemma 16, the existence of Hopf bifurcation implies a LC, while GP rules out the occurrence of Hopf bifurcation.

According to Corollary 7, a Hopf bifurcation appears only when preferences exhibit a compensation effect. Nevertheless, if this compensation effect becomes excessive and, therefore, the GP takes place, any Hopf bifurcation is ruled out. To explain this important result, we need to understand in economic terms how a Hopf bifurcation arises around the steady state.

First of all, according to Propositions 10 and 11, a weak compensation effect implies the existence of a LC, while an excessive compensation effect entails the occurrence of the GP. Now, Let the economy be at the steady state at time $t$ and assume an exogenous rise in the pollution level. Focus on the pollution accumulation process (12): the effect of this increase on the next-period pollution level is ambiguous. Indeed, (1) under the effect of the natural pollution absorption, the future pollution stock lowers, but (2) because of the compensation effect, the consumption demand increases, raising in turn the future pollution stock. Hence, the effect of a higher pollution level today on the pollution level tomorrow rests on the magnitude of the compensation effect. Under a weak compensation effect (compatible with the LC), (1) dominates (2), which means that a higher pollution level today implies a lower pollution level tomorrow giving rise to a endogenous fluctuations. Conversely, if the compensation effect becomes excessive (compatible with the GP), (2) dominates (1), which means that a higher pollution level today entails a higher pollution level tomorrow, preventing the occurrence of endogenous cycles in the end. Such an over-reaction, induced by the excessive compensation effect, neutralizes the restoring force.

\subsection{Simulation}

The previous section does not exclude the possibility of a Hopf bifurcation around the steady state under a sufficiently strong compensation effect. In addition, we know that a green consumption tax is unable to prevent endoge-

nous cycles (see section 9). But, does this tax modify the stability of the limit 
cycle emerging through the Hopf bifurcation? Unfortunately, the Jacobian matrix $J$ is uninformative. Higher-order informations are required. To address the important question of bifurcation criticity, we perform a numerical analysis.

We study the stability properties of the limit cycle when the economy is located on both side of the LC using the MATCONT package for MATLAB. We consider quarterly values $(\alpha, \rho$ and $\delta)$.

\begin{tabular}{lcccccccccc}
\hline \hline Parameter & $\alpha$ & $v$ & $A$ & $\rho$ & $\delta$ & $a$ & $b$ & $\gamma$ & $\varepsilon$ & $\xi$ \\
\hline Value & 0.33 & 1 & 1 & 0.01 & 0.025 & 0.1 & 0.05 & 0.1 & 2 & 4 \\
\hline \hline
\end{tabular}

We fix $\varepsilon$ to satisfy $\varepsilon_{c P}>0$. Finally, we choose $a, b$ and $\gamma$ to avoid excessive stationary values at the Hopf bifurcation point and correctly apply MATCONT.

Following Proposition 14 and calibration (37), a Hopf bifurcation generically occurs when $\eta=\eta^{H}=4.933$. According to proposition 10, a LC arises for $\eta \in(0,6)$, that is, a LC occurs at the steady state when $\eta=\eta_{H}$ (see Fig.1).

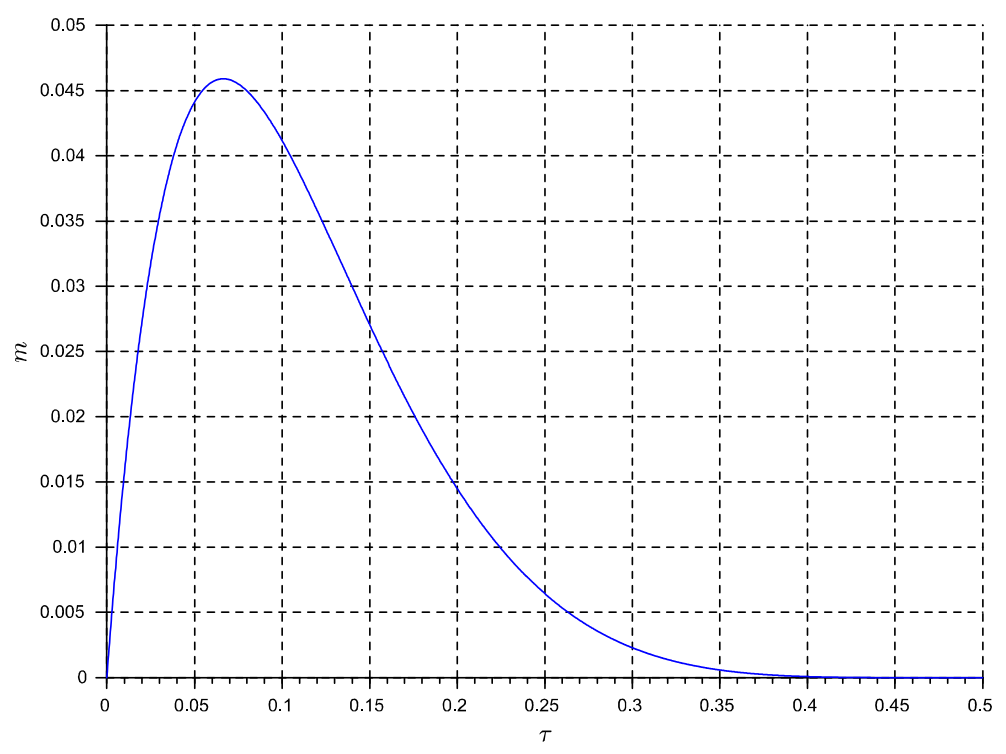

Fig.1 The Laffer Curve when $\eta=\eta_{H}$

The location of economy along the LC depends upon the level of the green consumption tax. First, assume that the economy lies on the upward-sloping branch of the LC. If $\tau=0.05$, the steady state values become

$$
(\lambda, k, P)=\left(1.2860 \times 10^{-2}, 28.471,0.39723\right)
$$

MATCONT detects and computes independently a Hopf bifurcation at $\eta=$ $4.9330157 \approx \eta_{H}$. The corresponding eigenvalues become:

$$
\begin{aligned}
& \lambda_{1}=0.0122273 i=-\lambda_{2} \\
& \lambda_{3}=0.156651
\end{aligned}
$$


$\lambda_{1}$ and $\lambda_{2}$ are purely imaginary conjugated eigenvalues (with zero real part) capturing the emergence of a limit cycle near the steady state. The stability of the limit cycle depends upon the sign of the first Lyapunov coefficient $\left(l_{1}\right)$ when the dynamic system undergoes a Hopf bifurcation. Computing the coefficient with MATCONT, we find $l_{1}=-5.279860 * 10^{-6}$. Since $l_{1}<0$, the Hopf bifurcation is supercritical and, thus, the limit cycle is stable (See Fig. 2).

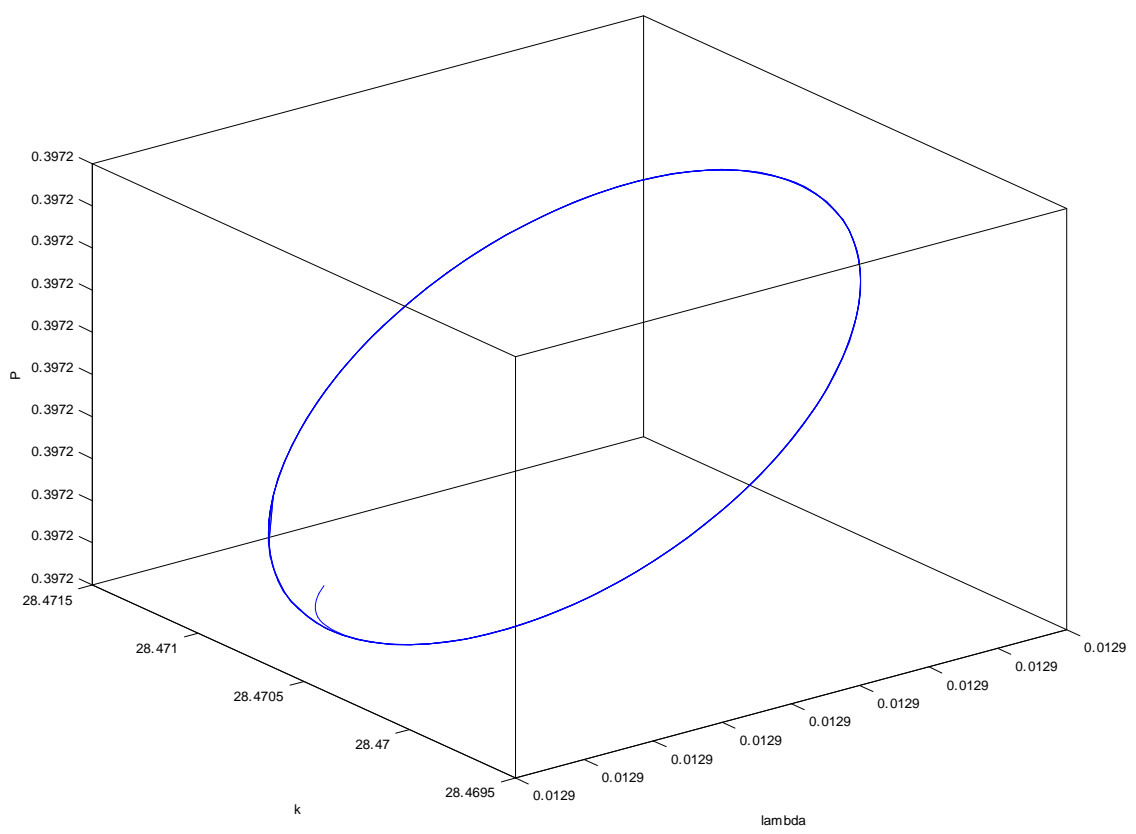

Fig.2 The stable limit cycle for $\tau=0.05$

Now, let the economy lie on the downward-sloping branch of the LC. Fix, for instance, $\tau=0.1$. The steady state becomes:

$$
(\lambda, k, P)=\left(7.3349 \times 10^{-4}, 28.471,0.16471\right)
$$

The critical Hopf bifurcation value does not change: $\eta=4.9330161 \approx \eta_{H}$ (it is independent of $\tau$ ). The corresponding eigenvalues are also the same than (38) and (39) (indeed $T, S$ and $D$ are also independent on $\tau$ : see expressions (32) to (34)). The first Lyapunov coefficient remains negative $l_{1}=-5.280228 * 10^{-6}<$ 0 . Therefore, the Hopf bifurcation remains supercritical and the limit cycle stable (see Fig.3). 


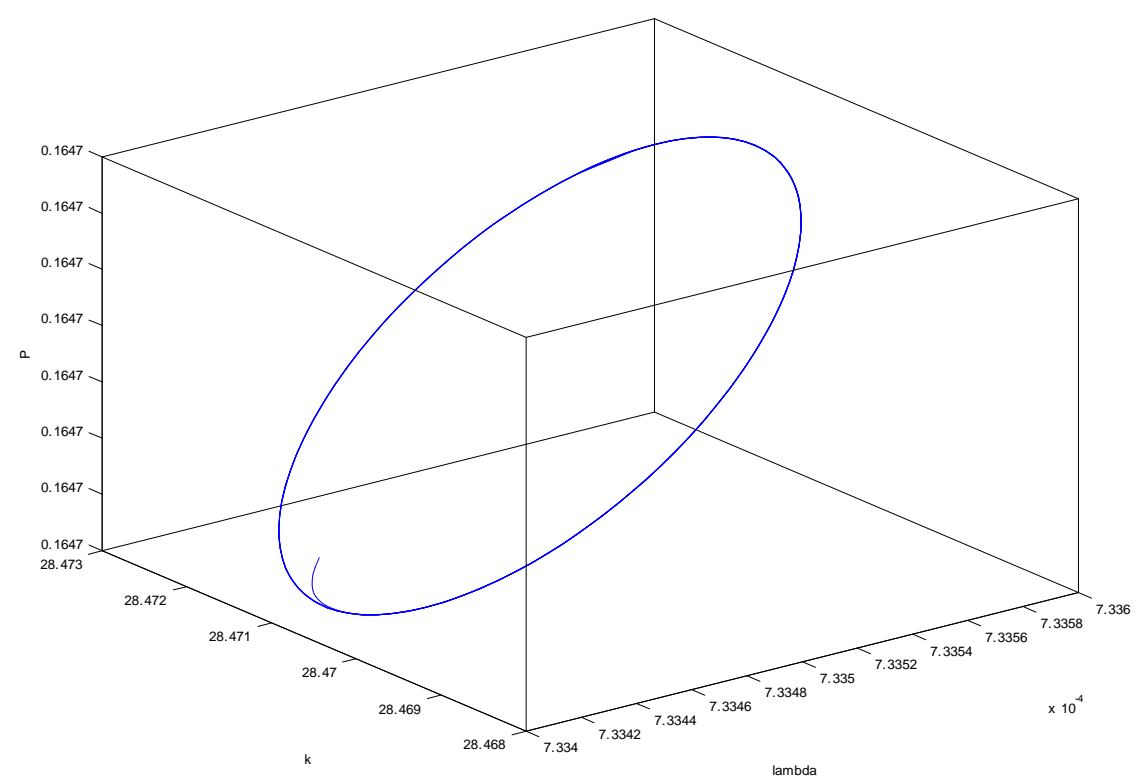

Fig. 3 The stable limit cycle for $\tau=0.1$

These numerical simulations shows that the location of the economy along the LC does not affect the stability of the limit cycle.

\section{Conclusion}

We have considered a Ramsey model with endogenous labor supply, where pollution increases the consumption demand through a compensation effect. We have seen that, in the long run, a weak compensation effect leads to a LC while a strong compensation effect leads to a GP. Of course, the incompatibility of these sufficient conditions suggests that LC and the GP are mutually exclusive. ${ }^{4}$

We have also shown that taxation is welfare-improving beyond a critical point: (1) under a weak compensation effect (sufficient for a LC) if households over-evaluate the environmental quality or (2) under a strong compensation effect (sufficient for the GP) if households under-evaluate the environmental quality.

Studying the local stability properties, we have found that the occurrence of limit cycles implies the existence of a LC but they are ruled out in the case of GP.

Our theoretical analysis has been complemented by a numerical simulation showing the supercriticity (stability) of the limit cycles along both the branches of the LC.

\footnotetext{
${ }^{4}$ Nevertheless, we observe that these conditions are only sufficient and the mutual exclusion is not automatic.
} 


\section{Appendix}

\section{Proof of Proposition 1}

The consumer's Hamiltonian function writes

$$
\tilde{H} \equiv e^{-\rho t}[u(c, P)-v(l)]+\tilde{\lambda}[(r-\delta) h+w l-(1+\tau) c]
$$

The first-order conditions are given by $\partial \tilde{H} / \partial \tilde{\lambda}=(r-\delta) h+w l-(1+\tau) c=\dot{h}$, $\partial \tilde{H} / \partial h=(r-\delta) \tilde{\lambda}=-\tilde{\lambda}^{\prime}, \partial \tilde{H} / \partial c=e^{-\rho t} u_{c}-(1+\tau) \tilde{\lambda}=0$ and $\partial \tilde{H} / \partial l=$ $-e^{-\rho t} v_{l}+w \tilde{\lambda}=0$ jointly with the transversality condition $\lim _{t \rightarrow \infty} \tilde{\lambda} h=0$. Setting $\lambda \equiv e^{\rho t} \tilde{\lambda}$, we find $\dot{\lambda}-\rho \lambda=e^{\rho t} \tilde{\lambda}^{\prime}$ and, therefore, $\lambda(r-\delta-\rho)=-\dot{\lambda}$. Finally, we obtain the budget constraint $\dot{h}=(r-\delta) h+w l-(1+\tau) c$, now binding.

Proof of Proposition 3

$\mu$ is a continuous function. Boundary conditions (19) ensures positive solutions $P^{*}$ of equation (18). Consider equation (18). If a steady state exists, the monotonicity of $\mu(\theta>0)$ jointly with the boundary conditions (19) ensures also the uniqueness of the steady state. More explicitly, we obtain

$$
\theta=\left(\frac{\lambda}{c} \frac{\partial c}{\partial \lambda}-\frac{\lambda}{l} \frac{\partial l}{\partial \lambda}\right) \frac{P}{\lambda} \frac{d \lambda}{d P}+\frac{P}{c} \frac{\partial c}{\partial P}=1-\frac{\varepsilon_{c c}+\varepsilon_{c P}}{\varepsilon_{l l}}
$$

Since $\varepsilon_{l l}>0, \varepsilon_{c P}<-\varepsilon_{c c}$ implies $\theta>0$.

\section{Proof of Proposition 4}

The tax elasticity of welfare function is given by

$$
\frac{\tau \tilde{W}^{\prime}(\tau)}{\tilde{W}(\tau)}=\frac{u}{u-v}\left[\varepsilon_{c} \frac{\tau \tilde{c}^{\prime}(\tau)}{\tilde{c}(\tau)}+\varepsilon_{P} \frac{\tau \tilde{P}^{\prime}(\tau)}{\tilde{P}(\tau)}\right]-\frac{v}{u-v} \varepsilon_{l} \frac{\tau \tilde{l}^{\prime}(\tau)}{\tilde{l}(\tau)}
$$

Replacing (21), (22) and (23), we find

$$
\frac{\tau \tilde{W}^{\prime}(\tau)}{\tilde{W}(\tau)}=\frac{\left[u\left(\varepsilon_{c}+\varepsilon_{P}\right)-v \varepsilon_{l}\right] \pi(\tau)-u \varepsilon_{P} \frac{\gamma \tau}{b-\gamma \tau}-v \varepsilon_{l} \frac{\tau}{1+\tau}}{u-v}
$$

(40) also writes

$$
\frac{\tau \tilde{W}^{\prime}(\tau)}{\tilde{W}(\tau)}=\frac{\left[u\left(\varepsilon_{c}+\varepsilon_{P}\right)-l v_{l}\right] \pi(\tau)-u \varepsilon_{P} \frac{\gamma \tau}{b-\gamma \tau}-l v_{l} \frac{\tau}{1+\tau}}{u-v}
$$

Replacing $v_{l}=w u_{c} /(1+\tau)$ (equation (6)), we find

$$
\frac{\tau \tilde{W}^{\prime}(\tau)}{\tilde{W}(\tau)}=\frac{u}{u-v}\left[\left(\varepsilon_{c}+\varepsilon_{P}-\frac{w}{1+\tau} \frac{l}{c} \varepsilon_{c}\right) \pi(\tau)-\varepsilon_{P} \frac{\gamma \tau}{b-\gamma \tau}-\frac{w}{1+\tau} \frac{l}{c} \varepsilon_{c} \frac{\tau}{1+\tau}\right]
$$

In addition, we obtain from (16)

$$
\frac{l}{c}=\frac{1+\tau}{\rho k+w}
$$


This implies:

$$
\frac{\tau \tilde{W}^{\prime}(\tau)}{\tilde{W}(\tau)}=\frac{u}{u-v}\left[\pi(\tau)\left(\varepsilon_{P}+\varepsilon_{c} \frac{\rho k}{\rho k+w}\right)-\varepsilon_{P} \frac{\gamma \tau}{b-\gamma \tau}-\varepsilon_{c} \frac{\tau}{1+\tau} \frac{w}{\rho k+w}\right]
$$

Since

$$
\frac{\rho k}{\rho k+w}=\frac{\alpha \rho}{(1-\alpha) \delta+\rho}
$$

with $\alpha=\alpha(k)=\alpha\left(r^{-1}(\rho+\delta)\right)$, but independent of $\tau$, we obtain

$$
\frac{\tau \tilde{W}^{\prime}(\tau)}{\tilde{W}(\tau)}=\frac{u}{u-v}\left[\pi(\tau)\left(\varepsilon_{P}+\varepsilon_{c} \frac{\alpha \rho}{(1-\alpha) \delta+\rho}\right)-\varepsilon_{P} \frac{\gamma \tau}{b-\gamma \tau}-\varepsilon_{c} \frac{\tau}{1+\tau} \frac{(1-\alpha)(\delta+\rho)}{(1-\alpha) \delta+\rho}\right]
$$

We observe that, $u-v=\rho \tilde{W}(\tau)$. Thus,

$\tilde{W}^{\prime}(\tau)=\frac{u \varepsilon_{c}}{\rho \tau}\left[\pi(\tau)\left(\frac{\varepsilon_{P}}{\varepsilon_{c}}+\frac{\alpha \rho}{(1-\alpha) \delta+\rho}\right)-\frac{\varepsilon_{P}}{\varepsilon_{c}} \frac{\gamma \tau}{b-\gamma \tau}-\frac{\tau}{1+\tau} \frac{(1-\alpha)(\delta+\rho)}{(1-\alpha) \delta+\rho}\right]$

Notice that $u \varepsilon_{c}>0$. Then, $\tilde{W}^{\prime}(\tau)>0$ if and only if

$$
\pi(\tau)\left(\frac{\varepsilon_{P}}{\varepsilon_{c}}+\frac{\alpha \rho}{(1-\alpha) \delta+\rho}\right)>\frac{\varepsilon_{P}}{\varepsilon_{c}} \frac{\gamma \tau}{b-\gamma \tau}+\frac{\tau}{1+\tau} \frac{(1-\alpha)(\delta+\rho)}{(1-\alpha) \delta+\rho}
$$

\section{Proof of Proposition 6}

Necessity In a three-dimensional dynamic system, we require at the bifurcation value: $\lambda_{1}=i b=-\lambda_{2}$ with no generic restriction on $\lambda_{3}$ (see Bosi and Ragot (2011) or Kuznetsov (1998) among others). The characteristic polynomial of $J$ is given by: $P(\lambda)=\left(\lambda-\lambda_{1}\right)\left(\lambda-\lambda_{2}\right)\left(\lambda-\lambda_{3}\right)=\lambda^{3}-T \lambda^{2}+S \lambda-D$. Using $\lambda_{1}=i b=-\lambda_{2}$, we find $D=b^{2} \lambda_{3}, S=b^{2}, T=\lambda_{3}$. Thus, $D=S T$ and $S>0$.

Sufficiency In the case of a three-dimensional system, one eigenvalue is always real, the others two are either real or nonreal and conjugated. Let us show that, if $D=S T$ and $S>0$, these eigenvalues are nonreal with zero real part and, hence, a Hopf bifurcation generically occurs.

We observe that $D=S T$ implies

$$
\lambda_{1} \lambda_{2} \lambda_{3}=\left(\lambda_{1} \lambda_{2}+\lambda_{1} \lambda_{3}+\lambda_{2} \lambda_{3}\right)\left(\lambda_{1}+\lambda_{2}+\lambda_{3}\right)
$$

or, equivalently,

$$
\left(\lambda_{1}+\lambda_{2}\right)\left[\lambda_{3}^{2}+\left(\lambda_{1}+\lambda_{2}\right) \lambda_{3}+\lambda_{1} \lambda_{2}\right]=0
$$

This equation holds if and only if $\lambda_{1}+\lambda_{2}=0$ or $\lambda_{3}^{2}+\left(\lambda_{1}+\lambda_{2}\right) \lambda_{3}+\lambda_{1} \lambda_{2}=0$. Solving this second-degree equation for $\lambda_{3}$, we find $\lambda_{3}=-\lambda_{1}$ or $-\lambda_{2}$. Thus, (41) holds if and only if $\lambda_{1}+\lambda_{2}=0$ or $\lambda_{1}+\lambda_{3}=0$ or $\lambda_{2}+\lambda_{3}=0$. Without loss of generality, let $\lambda_{1}+\lambda_{2}=0$ with, generically, $\lambda_{3} \neq 0$ a real eigenvalue. Since $S>0$, we have also $\lambda_{1}=-\lambda_{2} \neq 0$. We obtain $T=\lambda_{3} \neq 0$ and $S=D / T=\lambda_{1}$ 
$\lambda_{2}=-\lambda_{1}^{2}>0$. This is possible only if $\lambda_{1}$ is nonreal. If $\lambda_{1}$ is nonreal, $\lambda_{2}$ is conjugated, and, since $\lambda_{1}=-\lambda_{2}$, they have a zero real part.

\section{Proof of Proposition 9}

Necessity In the real case, we obtain $D=\lambda_{1} \lambda_{2} \lambda_{3}<0, S=\lambda_{1} \lambda_{2}+\lambda_{1} \lambda_{3}+$ $\lambda_{2} \lambda_{3}>0$ and $T=\lambda_{1}+\lambda_{2}+\lambda_{3}<0$.

Sufficiency We want to prove that, if $D, T<0$ and $S>0$, then $\lambda_{1}, \lambda_{2}, \lambda_{3}<$ 0 . Notice that $D<0$ implies $\lambda_{1}, \lambda_{2}, \lambda_{3} \neq 0$.

$D<0$ implies that at least one eigenvalue is negative. Let, without loss of generality, $\lambda_{3}<0$. Since $\lambda_{3}<0$ and $D=\lambda_{1} \lambda_{2} \lambda_{3}<0$, we have $\lambda_{1} \lambda_{2}>0$. Thus, there are two subcases: (1) $\lambda_{1}, \lambda_{2}<0$, (2) $\lambda_{1}, \lambda_{2}>0$. If $\lambda_{1}, \lambda_{2}>0, T<0$ implies $\lambda_{3}<-\left(\lambda_{1}+\lambda_{2}\right)$ and, hence,

$$
S=\lambda_{1} \lambda_{2}+\left(\lambda_{1}+\lambda_{2}\right) \lambda_{3}<\lambda_{1} \lambda_{2}-\left(\lambda_{1}+\lambda_{2}\right)^{2}=-\lambda_{1}^{2}-\lambda_{2}^{2}-\lambda_{1} \lambda_{2}<0
$$

a contradiction. Then, $\lambda_{1}, \lambda_{2}<0$.

\section{Proof of Proposition 10}

Consider (21). Because the second-order elasticities are constant with respect to $\tau$, we find

$$
\pi^{\prime}(\tau)=\frac{\pi(\tau)}{\tau(1+\tau)}+\frac{\tau}{1+\tau} \frac{\gamma(b+\gamma)}{(b-\gamma \tau)^{2}} \frac{\varepsilon_{c P}}{\varepsilon_{c c}+\varepsilon_{c P}-\varepsilon_{l l}}
$$

We observe that $\varepsilon_{c P}<\varepsilon_{l l}-\varepsilon_{c c}$ is equivalent to $\eta(\varepsilon-1)<\varepsilon+\xi$. Since $0<\eta(\varepsilon-1)<\varepsilon+\xi$ jointly with $\tau \in(0, b / \gamma)$ (that is $P>0$, see equation (17)), we have $\pi(\tau)<0$ with $\pi^{\prime}(\tau)<0$ (monotonicity). In addition:

$$
\pi(0)=0 \text { and } \lim _{\tau \rightarrow(b / \gamma)^{-}} \pi(\tau)=-\infty
$$

Let $\tau^{*} \in(0, b / \gamma)$ be solution of $\pi(\tau)=-1$. Because of continuity, monotonicity and boundary conditions, this solution exists and is unique.

We observe that

$$
\frac{\tau}{m} \frac{\partial m}{\partial \tau}=1+\pi(\tau)>0 \Leftrightarrow \pi(\tau)>-1 \Leftrightarrow \tau<\tau^{*}
$$

Therefore, the function $m=m(\tau)$ is concave with a maximum at $\tau=\tau^{*}$.

Proof of Proposition 13

Consider (21) and replace parametric expressions (28) to obtain

$$
\pi(\tau)=\frac{\eta(\varepsilon-1) \frac{\gamma \tau}{b-\gamma \tau}+(1+\xi) \frac{\tau}{1+\tau}}{\eta(\varepsilon-1)-\varepsilon-\xi}
$$

(21), (22) and (23) become respectively

$$
\frac{\tau \tilde{c}^{\prime}(\tau)}{\tilde{c}(\tau)}=\pi(\tau), \frac{\tau \tilde{l}^{\prime}(\tau)}{\tilde{l}(\tau)}=\pi(\tau)+\frac{\tau}{1+\tau}, \frac{\tau \tilde{P}^{\prime}(\tau)}{\tilde{P}(\tau)}=\pi(\tau)-\frac{\gamma \tau}{b-\gamma \tau}
$$


According to Proposition 4, $\tau$ has a positive effect on welfare if and only if

$$
\frac{\eta(\varepsilon-1) \frac{\gamma(1+\tau)}{b-\gamma \tau}+(1+\xi)}{\eta(\varepsilon-1)-\varepsilon-\xi}\left[\frac{\alpha \rho}{(1-\alpha) \delta+\rho}-\eta\right]>\frac{(1-\alpha)(\delta+\rho)}{(1-\alpha) \delta+\rho}-\eta \frac{\gamma(1+\tau)}{b-\gamma \tau}
$$

Simply remark that the RHS of 43 is always negative when $\tau>\tau^{*}$. In addition, (1) and (2) introduce parametric restrictions to ensure that the LHS of 43 is positive.

\section{References}

[1] Bosi S. and D. Desmarchelier (2015). Limit cycles under a negative effect of pollution on consumption demand: the role of an environmental Kuznets curve. Documents de recherche 15-04, Centre d'Études des Politiques Économiques (EPEE).

[2] Bosi S., D. Desmarchelier and L. Ragot (2015). Pollution effects on labor supply and growth. International Journal of Economic Theory 11, 371-388.

[3] Bosi S. and L. Ragot (2011). Introduction to discrete-time dynamics. CLUEB, Bologna.

[4] Eichner, T., and Pethig, R. (2011). Carbon leakage, the green paradox, and perfect future markets. International Economic Review 52, 767-805.

[5] Fernandez E., R. Pérez and J. Ruiz (2012). The environmental Kuznets curve and equilibrium indeterminacy. Journal of Economic Dynamics $\mathbb{B}$ Control 36, 1700-1717.

[6] Gerlagh R. (2011). Too much oil. CESifo Economic Studies 57, 79-102.

[7] Grafton R.Q., T. Kompas and N. Van Long (2012). Substitution between biofuels and fossil fuels: is there a Green Paradox? Journal of Environmental Economics and Management 64, 328-341.

[8] Heal G. (1982). The use of common property resources. In Explorations in Natural Resource Economics, The Johns Hopkins University Press for Resources for the Future, Baltimore.

[9] Ibn Khaldun (1377). The Muqaddimah: An Introduction to History. Translated by F. Rosenthal (1989), Bollingen series, Princeton University Press.

[10] Ireland P. (1994). Supply-side economics and endogenous growth. Journal of Monetary Economics 33, 559-571.

[11] Itaya J.-I. (2008). Can environmental taxation stimulate growth? The role of indeterminacy in endogenous growth models with environmental externalities. Journal of Economic Dynamics \& Control 32, 1156-1180. 
[12] Kuznetsov Y. (1998). Elements of Applied Bifurcation Theory. Springer, Applied Mathematical Sciences, vol. 112.

[13] Michel P. and G. Rotillon (1995). Disutility of pollution and endogenous growth. Environmental and Resource Economics 6, 279-300.

[14] Nourry C., T. Seegmuller and A. Venditti (2013). Aggregate instability under balanced-budget consumption taxes: a re-examination. Journal of Economic Theory 148, 1977-2006.

[15] Schmitt-Grohé S. and M. Uribe. (1997). Balanced-Budget Rules, Distortionary Taxes, and Aggregate Instability. Journal of Political Economy 105, 976-1000.

[16] Sinn H-W. (2008). Public policies against global warming: a supply side approach. International Tax and Public Finance 15, 360-394.

[17] Sinn H.W. (2012). The Green Paradox. MIT Press,Cambridge.

[18] Trabandt M. and H. Uhlig. (2011). The Laffer curve revisited. Journal of Monetary Economics 58, 305-327.

[19] Van der Ploeg F and C. Withagen. (2012). Is there really a Green Paradox ? Journal of Environmental Economics and Management 64,342-363.

[20] Van der Meijden G., F. Van der Ploeg and C. Withagen. (2015). International capital markets, oil producers and the Green Paradox. European Economic Review 76, 275-297.

[21] Wanniski J. (1978). The Way the World Works. 4th edition (1998), Regnery Publishing. 